\title{
Trauma ocular aberto: características de casos atendidos no complexo Hospitalar Padre Bento de Guarulhos
}

\author{
Ocular open trauma:characteristics of admitted cases at the Padre Bento Hospital \\ of Guarulhos, $S P$
}

Mônica Weyll'

Regina Cele Silveira²

Nilson Lopes da Fonseca Júnior ${ }^{3}$

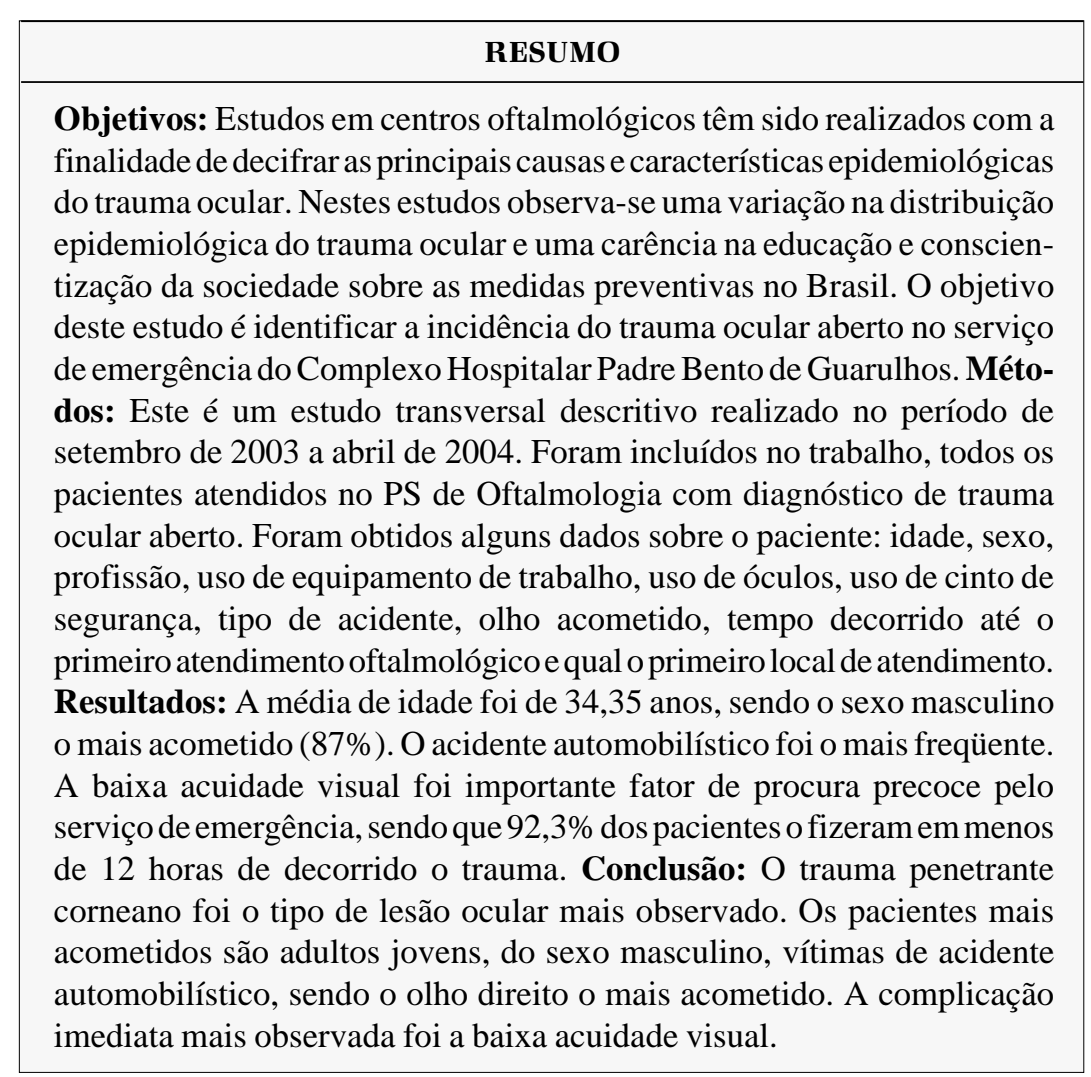

Descritores: Ferimentos oculares penetrantes/epidemiologia; Serviço hospitalar de emergência; Córnea/lesões; Acidentes de trânsito (CHPB) de Guarulhos (SP).

${ }^{1}$ Residente do segundo ano de Oftalmologia do Complexo Hospitalar Padre Bento (CHPB). Guarulhos (SP).

${ }^{2}$ Chefe do Setor de Glaucoma do CHPB. Guarulhos (SP). ${ }^{3}$ Chefe do Setor de Órbita/Plástica Ocular do CHPB. Guarulhos (SP).

Endereço para correspondência: Mônica Weyll. Rua Afonso Celso, 1000 - Apto. 54 - São Paulo (SP) CEP 04119-060

E-mail: mmpweyll@yahoo.com.br

Recebido para publicação em 02.08.2004

Versão revisada recebida em 26.02.2005

Aprovação em 12.04.2005

Nota Editorial: Após concluída a análise do artigo sob sigilo editorial e com a anuência do Dr. Christiano Fausto Barsante sobre a divulgação de seu nome como revisor, agradecemos sua participação neste processo.

\section{INTRODUĈ̣̃O}

O trauma ocular aberto é definido como perda de continuidade da túnica externa ocular em toda a sua espessura ${ }^{(1-4)}$. A túnica externa ocular é constituída pela córnea e esclera ${ }^{(1-3)}$.

Estudos em centros oftalmológicos têm sido realizados com a finalidade de decifrar as principais causas do trauma ocular, bem como outras características epidemiológicas ${ }^{(2,4-29)}$. De modo geral, tem sido detectada uma freqüência alta de trauma ocular aberto em adultos jovens e no sexo masculino $^{(2,4-25)}$.

Nos Estados Unidos, dois grandes sistemas de registro atualmente rastreiam a ocorrência do trauma ocular em quase todo o Estado: o NETS (National Eye Trauma System) e o USEIR (United States Eye Injury Registry) ${ }^{(5-7,10)}$.
\end{abstract}


O NETS é um sistema que integra diversos centros de oftalmologia do Estado americano com a missão de melhorar o manejo dos pacientes com trauma ocular severo, bem como estimular a pesquisa neste assunto ${ }^{(2,5-6)}$. O critério para entrar nesse sistema é que o trauma seja aberto, acometendo córnea e/ou esclera ${ }^{(2)}$. O registro dos casos é feito voluntariamente pelo centro oftalmológico regional, que integra o consórcio NETS $^{(2,5)}$. Através deste sistema, é possível monitorar as causas e características do trauma elaborando um correto manejo do mesmo, através de tratamento adequado, bem como medidas educacionais voltadas para a sociedade s.5-6) $^{(25}$.

O USEIR é um sistema de dados como o NETS; é englobado por 39 dos 50 estados americanos ${ }^{(7,9-11)}$. O USEIR registra os dados do paciente no momento do atendimento e durante os seis meses de acompanhamento ${ }^{(10)}$. Seu critério de inclusão é a presença de lesão ocular severa resultando em perda funcional e/ou anatômica permanente e significante ${ }^{(10)}$. O estudo deste registro desde sua fundação em 1988 até 1998 tem revelado uma mudança na epidemiologia do trauma ocular, quanto ao local mais freqüente de ocorrência, no estado americano $^{(10)}$. Trabalhos realizados nos anos 80 demonstraram uma taxa de acidente de trabalho alta, comparado a outras categorias de local de acidente ${ }^{(7,9-11)}$. Nos anos 90 , começou a ocorrer uma substituição do trauma ocular por acidente de trabalho pelo trauma doméstico, como principal causa ${ }^{(10)}$. Isso tem ocorrido nos Estados Unidos, devido ao uso de óculos de proteção durante o trabalho ${ }^{(10)}$. Ao contrário de países subdesenvolvidos, como o Brasil, o acidente automobilístico não configura uma causa importante de trauma ocular aberto, provavelmente devido à conscientização da sociedade através de medidas preventivas e educacionais, como o uso do cinto de segurança $\mathrm{a}^{(7,9-11)}$.

O EIRA (The Eye Injury Registry of Alabama), sistema desenvolvido no estado do Alabama para uso dos centros oftalmológicos locais, tem como finalidade o registro dos casos de trauma ocular aberto severos ${ }^{(11)}$. O USEIR surgiu deste sistema para então ser integrado aos demais estados americanos consorciados ${ }^{(10)}$.

Outros estudos também foram realizados nos Estados Unidos com o objetivo de traçar o perfil epidemiológico do trauma ocular nestas regiões ${ }^{(7-9)}$. Todos estes estudos americanos, incluindo também o EIRA, foram importantes para a criação dos sistemas de registro do trauma aberto nos Estados Uni$\operatorname{dos}^{(2,5-6,10)}$.

No período de 1989 a 1991 foram registrados todos os casos de perfuração ocular atendidos na região oeste da Suécia $^{(26)}$. Em Israel, foi realizado um estudo prospectivo de 1981 a 1983 no qual além de avaliar a epidemiologia de tais traumas, observaram o tempo de internamento hospitalar dispendido por estes pacientes ${ }^{(27)}$.

Estudos do trauma ocular infantil mostram que a classe social na qual convive a criança e o nível de escolaridade dos pais influi na gravidade do trauma ${ }^{(29)}$. A baixa idade está relacionada a um pior prognóstico visual final ${ }^{(4)}$, sendo que de um modo geral, mais da metade das crianças com perfuração ocular evoluem com visão final maior ou igual a 20/200 ${ }^{(4,28-29)}$.

O trauma ocular aberto é um problema de saúde pública, acometendo, em sua maioria, adultos jovens ${ }^{(12-18,27)}$. Conseqüentemente, as repercussões social e econômica são grandes já que está associado à incapacidade temporária ou mesmo permanente do olho acometido ${ }^{(12-15,27)}$.

As causas de trauma ocular variam em sua freqüência de acordo com as regiões estudadas e a época em que houve implementação de medidas preventivas aos diversos tipos de acidentes $^{(13-16)}$, como, por exemplo, os acidentes automobilísticos e o uso do cinto de segurança.

No Brasil não existe um sistema unificado de registro de trauma ocular, como se pode inferir pela literatura nacional sobre o tema ${ }^{(12-17,19-25)}$. Os estudos são realizados em diversos centros oftalmológicos com o objetivo de traçar o perfil epidemiológico do trauma ocular no país ${ }^{(12-17,19-25)}$. Estes estudos mostraram uma variação quanto à distribuição epidemiológica do trauma ocular de acordo com o local do estudo e o ano em que o mesmo foi realizado ${ }^{(12-17,19-25)}$. O que existe em comum entre eles é a constatação do quanto é carente a educação e conscientização da sociedade sobre as medidas preventivas relacionadas ao tema ${ }^{(12-17,19-25)}$.

Estudos no Brasil, demonstraram uma alta taxa de trauma ocular aberto em pacientes que sofreram acidentes automobilísticos e não estavam usando o cinto de segurança, bem como em passageiros acomodados no banco dianteiro ${ }^{(21,23-24)}$. O uso do cinto evita o choque do corpo com o pára-brisa no momento do impacto ${ }^{(21,23-24)}$.

Embora a medicina tenha avançado nos últimos anos em termos de diagnóstico e tratamento, o trauma ocular continua sendo uma importante causa de cegueira ${ }^{(7-11,20)}$. Medidas preventivas constituem um importante meio de ação na tentativa de reduzir a incidência de traumas oculares abertos ${ }^{(2,5-13,16,20,26-27)}$. O mau prognóstico visual vai depender não apenas das medidas preventivas implementadas, como também da qualidade e tempo de atendimento do paciente, gravidade do trauma, complicações imediatas e seguimento nas consultas subseqüentes ${ }^{(7-11,14-16,20,26-27)}$.

Existem alguns sinais, sugestivos ou diagnósticos, que vão guiar o oftalmologista no diagnóstico do trauma ocular aberto $^{(3)}$.

São considerados sinais oculares sugestivos de trauma aberto: laceração profunda de pálpebra, laceração conjuntival, hemorragia conjuntival importante pós-trauma, adesão localizada íris-corneana, câmara anterior rasa, defeito iriano, hipotonia, defeito em cápsula cristaliniana, opacidade cristaliniana aguda, hemorragia e/ou ruptura retiniana ${ }^{(3)}$.

A exposição da úvea, vítreo e/ou retina, o teste de Seidel positivo, a observação direta de um corpo estranho intraocular e este visto no raio-X ou ultra-sonografia são considerados sinais diagnósticos de trauma ocular aberto ${ }^{(3)}$.

Diversos autores observaram a necessidade de uma nomenclatura universal para o trauma ocular devido às variações na descrição e conceito de um mesmo tipo de trauma ocu- 
$\operatorname{lar}^{(1,30)}$. Houve a tentativa de desenvolver um score, porém este apresentava critérios insuficientes para tanto ${ }^{(30)}$. Atualmente existe a classificação de BETT (Birmingham Eye Trauma Terminology) para classificar o trauma, porém ela ainda é controversa $^{(1)}$. O aperfeiçoamento deste sistema de classificação é importante para tornar o trauma ocular uma língua universal entre os oftalmologistas de todo o mundo ${ }^{(1)}$. "The Birmingham Eye Trauma Terminology" (BETT) classifica o trauma ocular como aberto quando o globo ocular apresenta ferimento que atinge toda a espessura de sua parede. O trauma ocular aberto pode ser subclassificado em: (1) Ruptura: ferimento do globo ocular em toda a sua espessura, causado por objeto rombo, não cortante. O impacto resulta num aumento momentâneo da pressão intra-ocular (PIO) e um mecanismo de lesão de dentro para fora; (2) Laceração: ferimento do globo ocular em toda a sua espessura, normalmente causado por objeto cortante. O ferimento ocorre no sítio do impacto através de um mecanismo de lesão de fora para dentro; (3) Ferimento penetrante: laceração simples do globo ocular, usualmente causada por objeto cortante; (4) Ferimento com corpo estranho intra-ocular (CEIO): objeto estranho retido, causando laceração de entrada; (5) Ferimento perfurante: duas lacerações em toda a espessura (entrada e saída) do globo ocular, normalmente causada por um objeto cortante ou tipo míssil ${ }^{(1)}$.

Este trabalho tem o objetivo de identificar algumas características do trauma ocular aberto no serviço de emergência do Complexo Hospitalar Padre Bento de Guarulhos.

\section{MÉTODOS}

Trata-se de um estudo descritivo transversal realizado no serviço de emergência do Complexo Hospitalar Padre Bento de Guarulhos/São Paulo, no período de setembro de 2003 a abril de 2004.

Foram inclusos na pesquisa, os pacientes atendidos no PS de oftalmologia com o diagnóstico de trauma ocular aberto que aderiram ao termo de consentimento.

O trabalho avaliou o paciente no momento do primeiro atendimento oftalmológico, não sendo considerado o retorno do mesmo ao serviço.

No momento da admissão foram obtidos alguns dados sobre o paciente: idade, sexo, profissão, uso de equipamento de proteção individual, uso de óculos, uso de cinto de segurança, tipo de acidente, olho acometido, tempo decorrido até o primeiro atendimento médico e qual o primeiro local de atendimento. A acuidade visual imediata foi avaliada através de tabela cortical. Procedeu-se então, ao exame oftalmológico detalhado de todas as estruturas do segmento anterior do olho acometido, na lâmpada de fenda, a fim de identificar a lesão ocular e suas complicações imediatas. Nos casos de pacientes com sinais sugestivos de trauma ocular aberto oculto, foram realizados tonometria com tonômetro de Goldman e mapeamento de retina para completar a investigação diagnóstica.

Após avaliação inicial, todos os pacientes foram submeti- dos à internação hospitalar, sendo prescrito antibioticoterapia endovenosa (gentamicina $80 \mathrm{mg}-8 / 8$ horas e cefalotina $1 \mathrm{~g}$ - 12/12 horas), curativo oclusivo não-compressivo do olho acometido e avaliação pré-operatória para cirurgia sob anestesia geral.

\section{RESULTADOS}

Foram atendidos 30 pacientes com trauma ocular aberto com idade variando entre 14 e 84 anos (média de 34,35 anos). A distribuição do trauma pela faixa etária pode ser observada no gráfico 1.

Vinte e cinco $(83,3 \%)$ pacientes eram do sexo masculino e 5 $(16,7 \%)$ do sexo feminino. Doze $(40 \%)$ eram da raça branca, 9 (30\%) da raça parda e $9(30 \%)$ da raça negra.

O tempo decorrido entre o acidente até o primeiro atendimento médico não especializado variou de 15 minutos a 3 dias, e o tempo até o atendimento médico oftalmológico no CHPB variou entre 2 horas e 10 dias (Tabela 1).

Os tipos de acidente mais freqüentes foram o automobilístico $(40 \%)$ e o ocupacional $(36,7 \%)$ (Tabela 2$)$.

Dentre os pacientes que sofreram acidente automobilístico apenas $3(25 \%)$ usavam o cinto de segurança.

Onze casos $(36,7 \%)$ decorreram de acidente de trabalho, sendo que nenhum dos pacientes estava em uso de equipamento de proteção individual (EPI) no momento do trauma.

Dos 30 pacientes estudados, $15(50 \%)$ apresentaram trauma ocular por objeto pontiagudo, $1(3,3 \%)$ por arma de fogo e $1(3,3 \%)$ por agressão física.

Apenas em dois pacientes $(6,7 \%)$ observou-se uso de óculos no momento do trauma.

O olho mais freqüentemente acometido foi o direito, em 17 pacientes $(53,1 \%)$, seguido pelo esquerdo em 11 casos $(34,4 \%)$ e por ambos os olhos em 2 casos $(6,3 \%)$.

O tipo de trauma mais freqüente, pela classificação de BETT, foi o penetrante, correspondendo a 53,1\% dos mesmos (Tabela 3).

Corpo estranho intra-ocular foi identificado apenas nos traumas por acidente de trabalho e automobilístico.

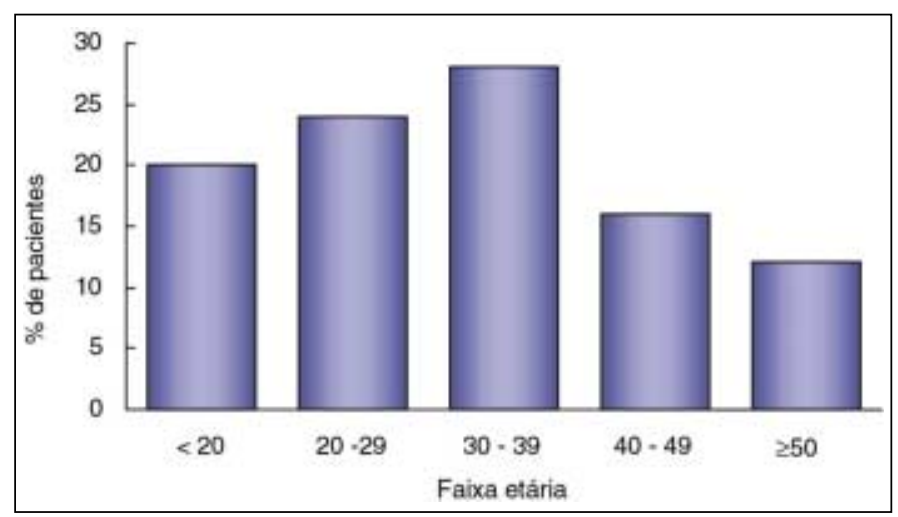

Gráfico 1 - Distribuição da faixa etária em pacientes com diagnóstico de trauma ocular aberto 


\begin{tabular}{|c|c|c|c|}
\hline \multirow[t]{2}{*}{ Variável } & Categoria & $\mathrm{n}$ & $\%$ \\
\hline & $\leq 12$ horas & 26 & 86,7 \\
\hline \multirow{3}{*}{$\begin{array}{l}\text { Tempo entre } o \text { acidente e } 0 \\
1^{\circ} \text { atendimento médico não especializado }\end{array}$} & $13 \mathrm{~h}$ a $24 \mathrm{~h}$ & 2 & 6,7 \\
\hline & $25 \mathrm{~h}$ a $48 \mathrm{~h}$ & 1 & 3,3 \\
\hline & $>48$ horas & 1 & 3,3 \\
\hline \multirow{4}{*}{ Local do $1^{\circ}$ atendimento } & CHPB (especializado) & 5 & 16,7 \\
\hline & Hospital municipal (não especializado) & 2 & 6,7 \\
\hline & Hospital geral (não especializado) & 8 & 26,7 \\
\hline & Posto de saúde (não especializado) & 15 & 50,0 \\
\hline \multirow{4}{*}{ Tempo até o CHPB (especializado) } & $\leq 12$ horas & 19 & 63,3 \\
\hline & $13 \mathrm{~h}$ a $24 \mathrm{~h}$ & 4 & 13,3 \\
\hline & $25 \mathrm{~h}$ a $48 \mathrm{~h}$ & 1 & 3,3 \\
\hline & $>48$ horas & 6 & 20,0 \\
\hline
\end{tabular}

No exame oftalmológico, $12(37,5 \%)$ pacientes com lesão córneo-escleral evoluíram com acuidade visual menor que 20/200, seguidos de $9(28,1 \%)$ com lesão corneana e $5(15,6 \%)$ com lesão escleral. Oitenta e quatro por cento dos pacientes avaliados evoluíram imediatamente com visão menor que 20/200 (Tabela 4).

Quanto ao tipo de trauma no tecido ocular, observou-se que 13 pacientes $(40,6 \%)$ apresentaram laceração córneoescleral, $12(37,5 \%)$ laceração apenas corneana e 7 (21,9\%) laceração escleral. A maioria das lesões acometeu o segmento posterior, correspondendo a $63 \%$ casos.

Através do teste exato de Fisher, observamos que há associação entre a catarata traumática e o tempo de atendimento. As maiores porcentagens de catarata estão em menos de 12 horas e entre 13 a 24 horas de atendimento (Tabela 5). Não houve significância estatística entre baixa acuidade visual (BAV) e tipo do trauma pela classificação de BETT.

Observamos que há associação entre a extensão de corpo uveal e vítreo e a classificação de BETT. As maiores porcentagens desta complicação estão nos casos de ruptura do globo ocular (Tabela 6).

\section{DISCUSSÃO}

Este foi o primeiro trabalho realizado no Complexo Hospitalar Padre Bento com o intuito de traçar o perfil do trauma ocular aberto nos pacientes admitidos neste serviço.

O presente estudo apresentou como faixa etária mais freqüentemente acometida pelo trauma aberto entre 20 e 39 anos, e o sexo masculino o mais afetado, o que não difere da literatu$\mathrm{ra}^{(2,29)}$. Outros estudos mostram uma maior freqüência do trauma ocular aberto no sexo masculino com média etária de 33 $\operatorname{anos}^{(26)}$. Observou-se neste estudo que não existiram pacientes com menos 14 anos. Isto ocorreu porque o Complexo Hospitalar Padre Bento não apresenta ala pediátrica, podendo ser um viés de aferição do trabalho, já que estes pacientes provavelmente foram encaminhados a uma unidade pediátrica com serviço de oftalmologia.
O tipo de trauma mais comum neste estudo foi o automobilístico, seguido do ocupacional. Neste último, nenhum paciente estava em uso de equipamento de proteção individual (EPI). Analisando-se os estudos nacionais observa-se que a incidência de perfuração ocular em cidades grandes é aparentemente maior que em cidades de pequeno porte (12-13,15-17,19-20,24) $_{\text {. }}$ Nas cidades menores predominam o trauma ocular secundário

\begin{tabular}{lcc}
\multicolumn{3}{c}{ Tabela 2. Freqüências absolutas e relativas do tipo de acidente } \\
Tipo de acidente & $\mathbf{n}$ & $\%$ \\
Automobilístico & 12 & 40,0 \\
Ocupacional & 11 & 36,7 \\
Doméstico & 5 & 16,7 \\
Violência* & 2 & 6,7 \\
n= número de pacientes & & \\
*Agressão física, ferimento por arma branca, ferimento por arma de fogo
\end{tabular}

\begin{tabular}{lcc|}
\hline \multicolumn{3}{l}{ Tabela 3. Freqüências absolutas e relativas da classificação de BETT } \\
Tipo de trauma & $\mathbf{n}$ & $\%$ \\
Laceração com ferimento penetrante & 17 & 53,1 \\
Laceração com ferimento perfurante & 7 & 21,9 \\
Ruptura & 5 & 15,6 \\
Laceração com CEIO & 3 & 9,4 \\
BETT=Birmigham Eye Trauma Terminollogy; $n=$ número de olhos; CEIO=corpo \\
estranho intra-ocular
\end{tabular}

\begin{tabular}{lcc} 
Tabela 4. Freqüências absolutas e relativas da AV do olho acometido \\
Acuidade visual & Olho acometido \\
\cline { 2 - 3 } & $\mathbf{n}$ & $\%$ \\
$20 / 20$ até $20 / 60$ & 2 & 6,3 \\
$20 / 70$ até 20/200 & 3 & 9,4 \\
Conta dedos & 6 & 18,8 \\
Movimento de mãos & 9 & 28,1 \\
Percepção de luminosidade & 3 & 9,4 \\
Sem percepção de luminosidade & 9 & 28,1
\end{tabular}




\begin{tabular}{|c|c|c|c|c|c|c|c|c|c|}
\hline \multirow[b]{3}{*}{ Complicações } & \multicolumn{8}{|c|}{ Tempo até o CHBP (em horas) } & \multirow[b]{3}{*}{$\mathbf{p}^{*}$} \\
\hline & \multicolumn{2}{|c|}{$\leq 12$} & \multicolumn{2}{|c|}{13 a 24} & \multicolumn{2}{|c|}{25 a 48} & \multicolumn{2}{|c|}{$>48$} & \\
\hline & $\mathbf{n}$ & $\%$ & $\mathbf{n}$ & $\%$ & $\mathbf{n}$ & $\%$ & $\mathbf{n}$ & $\%$ & \\
\hline BAV & 15 & 78,9 & 4 & 100,0 & 0 & 0,0 & 4 & 66,7 & 0,150 \\
\hline Catarata traumática & 6 & 31,6 & 3 & 75,0 & 1 & 100,0 & 2 & 33,3 & 0,013 \\
\hline Extensão de corpo uveal e vítreo & 1 & 5,3 & 0 & 0,0 & 0 & 0,0 & 1 & 16,7 & 0,682 \\
\hline Três ou mais complicações & 10 & 52,6 & 4 & 100,0 & 1 & 100,0 & 5 & 83,3 & 0,252 \\
\hline
\end{tabular}

\begin{tabular}{|c|c|c|c|c|c|c|c|c|c|}
\hline \multirow[b]{3}{*}{ Complicações } & \multicolumn{8}{|c|}{ BETT } & \multirow[b]{3}{*}{$\mathbf{p}^{*}$} \\
\hline & \multicolumn{2}{|c|}{ CEIO } & \multicolumn{2}{|c|}{$\begin{array}{l}\text { Ferimento } \\
\text { penetrante }\end{array}$} & \multicolumn{2}{|c|}{$\begin{array}{l}\text { Ferimento } \\
\text { perfurante }\end{array}$} & \multicolumn{2}{|c|}{ Ruptura } & \\
\hline & $\mathbf{n}$ & $\%$ & $\mathbf{n}$ & $\%$ & $\mathbf{n}$ & $\%$ & $\mathbf{n}$ & $\%$ & \\
\hline BAV & 2 & 66,7 & 14 & 82,4 & 6 & 85,7 & 5 & 100,0 & 1,000 \\
\hline Catarata traumática & 2 & 66,7 & 9 & 52,9 & 2 & 28,6 & 0 & 0,0 & 0,477 \\
\hline Extensão de corpo uveal e vítreo & 0 & 0,0 & 1 & 5,9 & 0 & 0,0 & 2 & 40,0 & 0,023 \\
\hline Três ou mais complicações & 1 & 33,3 & 12 & 70,6 & 7 & 100,0 & 2 & 40,0 & 0,105 \\
\hline
\end{tabular}

a atividades de lazer ${ }^{(13)}$ ou doméstico ${ }^{(15)}$, e nos grandes centros, acidentes automobilísticos e a violência ${ }^{(12)}$. A literatura também mostra grande freqüência de trauma doméstico nos EUA $^{(2,7-11)}$. Existe relação entre o tipo de trauma ocular, a atividade profissional exercida pelo paciente e o estilo de vida da região onde vive ${ }^{(15)}$.

Em estudos sobre acidentes automobilísticos, o que se observa é a falta de conscientização quanto ao uso do cinto de segurança pela população ${ }^{(20-21,23)}$, e, atualmente, mesmo com campanhas em massa realizadas pelo governo federal e estadual pelo uso do cinto de segurança e com implementação de multas pela falta de uso do mesmo, encontrou-se, neste estudo, uma alta taxa de acidente automobilístico. Esse dado não diferiu de outros encontrados em estudos realizados há dez anos no Brasil ${ }^{(5,20-21,23)}$. O que pode ter contribuído para a alta taxa de pacientes com acidente automobilístico é o Complexo Hospitalar Padre Bento ser referência regional em politrauma e oftalmologia.

De um modo geral, a literatura tem mostrado uma acuidade visual inicial menor que 20/200 na maioria dos pacientes $^{(2,8,10-11,15-16,18)}$, o que não difere deste trabalho, cuja visão menor que 20/200 corresponde a $84 \%$ dos pacientes atendidos. Estes estudos sugerem o quanto é necessário um atendimento específico adequado e intervenção cirúrgica rápida para tentar melhorar o prognóstico visual final destes pacientes. O tempo decorrido entre o acidente e a retirada do corpo estranho intra-ocular foi fundamental para um prognóstico visual melhor ${ }^{(12)}$. No levantamento atual constataram-se apenas três casos com CEIO, e em todos eles a visão foi menor que $20 / 200$.
Este trabalho associou o trauma à esclera como o mais freqüentemente relacionado à acuidade visual imediata menor que 20/200. Outros estudos têm mostrado que o tipo de tecido está relacionado à gravidade da lesão ${ }^{(2)}$, e que existe relação entre acuidade visual final e o local da lesão, sendo de pior prognóstico lesões acometendo esclera ${ }^{(16)}$ e de melhor prognóstico lesões corneanas ${ }^{(8)}$. Porém, como o presente estudo descritivo, não se pode inferir que o trauma corneano tenha prognóstico melhor, pois na primeira avaliação este também esteve associado à baixa acuidade visual imediata e não foi avaliada acuidade visual final.

Observou-se que a baixa acuidade visual esteve implicada numa procura mais precoce pelo serviço de emergência, porém nenhum destes trabalhos analisa o tempo de atendimento após o trauma com o prognóstico visual. Um estudo retrospectivo brasileiro de 768 pacientes com perfuração ocular atendidos em São Paulo, observou-se que $40 \%$ dos casos foram atendidos em serviço de oftalmologia nas primeiras seis horas do trauma e $14 \%$ após dois dias ${ }^{(24)}$. Neste trabalho, $56,5 \%$ dos pacientes foram atendidos em até 12 horas decorridos do trauma, podendo-se inferir que a baixa acuidade precoce leva o paciente mais cedo ao serviço especializado de emergência.

O diagnóstico precoce e prognóstico visual pobre são medidas acuradas para determinar a severidade da lesão ${ }^{(11)}$. Existe uma melhora no prognóstico visual de pacientes que foram submetidos a exame e intervenção clínico-cirúrgica rapidamente $^{(10)}$. O mesmo não se observa nos casos de lesões muito graves do globo ocular, sendo estas medidas tomadas 
no intuito de prevenir outras complicações severas decorrentes do trauma ${ }^{(10)}$.

A alta frequiência de atendimento primário em serviços de saúde não especializado, principalmente em pronto atendimento foi também um achado importante deste estudo. Isto mostra o quanto é importante o médico plantonista de serviço de saúde não especializado saber encaminhar rapidamente o paciente com história de trauma ocular a um serviço especializado para um correto manejo do caso.

\section{CONCLUSÃO}

O trauma penetrante corneano foi o tipo de lesão ocular mais observado. Os pacientes mais acometidos são adultos jovens, do sexo masculino, vítimas de acidente automobilístico, sendo o olho direito o mais acometido. A complicação imediata mais observada foi a baixa acuidade visual.

\section{ABSTRACT}

Purpose: This study tends to identify the incidence of ocular open trauma at the emergency service of the "Complexo Hospitalar Padre Bento of Guarulhos, SP'. Methods: It is a descriptive transversal study performed in a period of four months at the "Complexo Hospitalar Padre Bento". All patients admitted at the emergency room with a diagnosis of open ocular trauma were included. After ophthalmologic examination, all patients hospitalized, with intravenous antibiotic therapy, occlusive tamper and preparation for surgery. Results: Twenty-three patients with ocular perforating trauma were attended. Mean age was 34.35 years, with males representing $87.00 \%$ of all cases. Motor vehicle accidents were the most frequent type of trauma with $43.50 \%$ of the patients. Visual acuity loss was an important predictor of looking for early medical emergency service, with $92.30 \%$ of the patients doing this in less than twelve hours. Conclusion: Penetrating corneal trauma was the most frequent. Open ocular trauma was more frequent in young men, victims of automobile accident. The right eye was the most affected. Low vision was the mostly observed immediate complication.

Keywords: Eye injuries, penetrating/epidemiology; Emergency service, hospital; Cornea/injuries; Accidents, Traffic

\section{REFERÊNCIAS}

1. Kuhn F, Morris R, Witherspoon D, Heimann K, Jeffers JB, Treister G. A standardized classification of ocular trauma. Ophthalmology. 1996;103(2): 240-3.

2. Parver LM, Dannenberg AL, Blacklow B, Fowler CJ, Brechner RJ, Tielsch JM. Characteristics and causes of penetrating eye injuries reported to the National Eye Trauma System Registry, 1985-91. Public Health Rep. 1993; 108(5):625-32.
3. Reder JRCL, Carvalho FLM. Urgências em oftalmologia. In: Petroianu A, editor Urgências clínicas e cirúrgicas. Rio de Janeiro: Guanabara; 2002. p.210-23.

4. Farr AK, Hairston RJ, Humayun MU, Marsh MJ, Pieramici DJ, MacCumber MW et al. Open globe injuries in children: a retrospective analysis. J Pediatr Ophthalmol Strabismus. 2001;38(2):72-7.

5. Parver LM. The national eye trauma system. Int Ophthalmol Clin. 1988;28(3): 203-5.

6. Feist RM, Farber MD. Ocular trauma epidemiology. Arch Ophthalmol. 1989; 107(4):503-4.

7. Glynn RJ, Seddon JM, Berlin BM. The incidence of eye injuries in New England adults. Arch Ophthalmol. 1988;106(6):785-9.

8. Liggett PE, Pince KJ, Barlow W, Ragen M, Ryan SJ. Ocular trauma in an urban population. Review of 1,132 cases. Ophthalmology. 1990;97(5):581-4.

9. Schein OD, Hibberd PL, Shingleton BJ, Kunzweiler T, Frambach DA, Seddon JM et al. The spectrum and burden of ocular injury. Ophthalmology. 1988;95(3):300-5.

10. May DR, Kuhn FP, Morris RE, Witherspoon CD, Danis RP, Matthews GP et al. [The epidemiology of serious eye injuries from the United States eye injury registry]. Graefe's Arch Clin Exp Ophthalmol. 2000;238(2):153-7. Germany.

11. White MF Jr, Morris R, Feist RM, Witherspoon CD, Helms HA Jr, John GR Eye injury: prevalence and prognosis by setting. South Med J. 1989;82(2):151-8.

12. Carani JCE, Machado CG, Gomi CF, Carvalho RMS. Ferimentos perfurantes oculares no Hospital das Clínicas da Faculdade de Medicina da Universidade de São Paulo. O que mudou nos últimos 27 anos. Arq Bras Oftalmol. 1999; 62(3):310-4.

13. Aragaki GN, Inada ET, Teixeira MF, Almeida Jr GC, Kashiwabuchi LK. Estudo Epidemiológico dos traumas oculares graves em um Hospital Universitário de São José do Rio Preto - SP. Arq Bras Oftalmol. 2003;66(4):473-6.

14. Alves MR, Kara José N. O trauma ocular como causa de cegueira. Rev Méd São Paulo. 1997;76(6):297-302.

15. Schellini AS, D'Aurea Filho JA, Padovani CR, Silva MRBM. Causas e características do trauma ocular perfurante em Botucatu - SP. Rev Bras Oftalmol. 1995;54(11):31-6.

16. Bordon AF, Sousa LB, Moraes NSB, Freitas D. Perfuração ocular: estudo de 473 casos. Arq Bras Oftalmol. 1994;57(1):62-5.

17. Cohen J, Carvalho RC, Romão E. Trauma ocular por acidente de trabalho em Manaus (AM). Rev Bras Oftalmol.1994;53(2):69-72.

18. Franzco RJC, Walker JC, Fraco HSN. Four-year review of open eye injuries at the Royal Adelaide Hospital. Clin Exp Ophthalmol. 2002;30(1):15-8.

19. Bernucci EA, Lopreto RCC, Rodrigues MLV. Traumatismos oculares em uma Unidade de Emergência. Rev Bras Oftalmol. 1993;52(6):407-11.

20. Alves MR, Kara José N, Prado Jr. J, Usuba FS, Onclinx TM, Marantes CR Ferimento perfurante ocular: 400 casos admitidos na Clínica Oftalmológica do Hospital das Clínicas da Faculdade de Medicina da Universidade de São Paulo. Arq Bras Oftalmol. 1995;58(5):342-5.

21. Kara José N, Alves MR, Sampaio MW, Bonanomi MTB.[Perforating wounds of the eyeball caused by automobile accidents]. Bol Oficina Sanit Panam. 1983;95(6):547-55. Spanish

22. Kara José N, Rangel FF, Barbosa NLM. Perfurações do globo ocular e ferimentos da face: necessidade de diagnóstico precoce. Arq Bras Oftalmol. 1982;45(2):66-9

23. Sampaio MW, Alves MR, Kara José N. Ferimentos perfurantes do globo ocular por acidentes automobilísticos: casos atendidos durante o ano de 1994. Rev Hosp Clin Fac Med Univ São Paulo. 1996;51(2):69-71.

24. Bonanomi MTBC, Alves MR, Kara José N, Souza Jr. NA. Ferimento perfurante de globo ocular em adultos. Arq Bras Oftalmol. 1980;43(3):81-7.

25. Kara José N, Alves MR, Oliveira PR. Como educar a população para a prevenção do trauma ocular. Arq Bras Oftalmol. 1992;55(4):160-2.

26. Byhr E. Perforating eye injuries in a western part of Sweden. Acta Ophthalmol (Copenh). 1994;72(1):91-7.

27. Koval R, Teller J, Belkin M, Romen M, Yanko L, Savir H. The Israeli Ocular Injuries Study. A nationwide collaborative study. Arch Ophthalmol. 1988;106(6):776-80.

28. Thompson CG, Kumar N, Billson FA, Martin F. The aetiology of perforating ocular injuries in children. Br J Ophthalmol. 2002;86(8):920-2.

29. Moreira Jr. CA, Debert-Ribeiro M, Belfort Jr R. Epidemiology study of eye injuries in brazilian children. Arch Ophthalmol. 1988;106(6):781-4.

30. Kuhn F, Maisiak R, Mann L, Mester V, Morris R, Witherspoon CD. The ocular trauma score (OTS). Ophthalmol Clin North Am. 2002;15(2):163-5. 